\title{
Total Nasal Reconstruction after Nasectomy in a Case of Massive Squamous Cell Carcinoma of the Inferior Nose
}

\section{Julius Hoehne* and Helmut Fischer}

Department of Facial Plastic Surgery, Centre of Plastic Stuttgart, Germany

*Corresponding author : Julius Hoehne, MD, Department of Facial Plastic Surgery, Centre of Plastic Stuttgart, Marienhospital Stuttgart, Boheimstr, Stuttgart, Germany, Tel: +49 7116489 8241; Fax: +49 7116489 8242; E-mail: julius.hoehne@vinzenz.de

Received date: January 02, 2016; Accepted date: February 26, 2016; Published date: March 04, 2016

Copyright: (C) 2016 Julius $\mathrm{H}$, et al. This is an open-access article distributed under the terms of the Creative Commons Attribution License, which permits unrestricted use, distribution, and reproduction in any medium, provided the original author and source are credited.

\begin{abstract}
Total nasal reconstruction after radical nasectomy remains one of the most challenging tasks plastic surgeons face. In our study, the case of a 55-yearold- man who underwent radical nasectomy and subsequent total nasal reconstruction using autologous tissues is described. The question whether to continue the use of a nasal prosthesis or whether to reconstruct the nose should be carefully addressed in each individual case, and depends on patient as well as surgeon factors. If the patient is willing and fit to undergo major reconstructive surgery of the nose, autologous reconstruction can be successfully carried out by an experienced team, and a result superior to a prosthetic solution can be expected.
\end{abstract}

\section{Introduction}

Patients and attending surgeons, confronted with radical nasectomy defects, face a challenging choice: whether to continue the use of a nasal prosthesis, or whether to reconstruct the nose using autologous tissues, an extremely challenging and demanding series of operations for both, surgeon and patient $[1,2]$. Various aspects need to be taken into consideration before taking a decision, first and foremost whether it is the patient's wish to move away from a prosthesis which he or she might already be using. Second, as in all nasal reconstruction, form and function remain the most important determinants in such a challenging decision. Therefore, a total reconstruction of the nose should only be considered if an outcome equivalent to prosthesis in terms of form and function can be expected.

Possible disadvantages of prostheses such as regular need for cleaning, or loosening, need to be weighed against a possibly disastrous outcome of reconstructive surgery if done in a less experienced center. Furthermore, the mental and physical state and age of the patient play a critical role, as clearly not every patient is suitable for four to six major surgeries, mentally and physically.

\section{Case Report}

A 55-year-old man presented to our department for a second opinion in July 2009 with a massive squamous cell carcinoma (SCC) of the inferior nose, including the septum, columella and the tip of the nose (Figure 1).
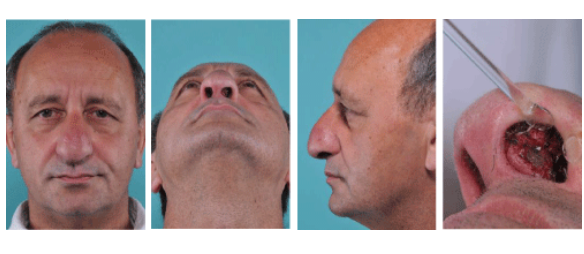

Figure 1: Initial presentation. A 55-year-old man presenting with massive SCC of the inferior nose.

We recommended radical tumor resection, followed by temporary supply with a nasal prosthesis for two years to detect early recurrence, and subsequent total autologous reconstruction of the nose (Figure 1). Radical tumor resection and implantation of magnet-carrying implants to the bony dorsum and right bony nasal floor was then performed by a nearby university hospital in August 2009. Staging was done including ultrasound of the neck and abdomen, as well as abdominal CT and MRI of the skull. No metastases were detected, yielding a T4N0M0 R0 situation. The patient was provided with a silicone prosthesis for two years to detect early recurrence (Figure 2).

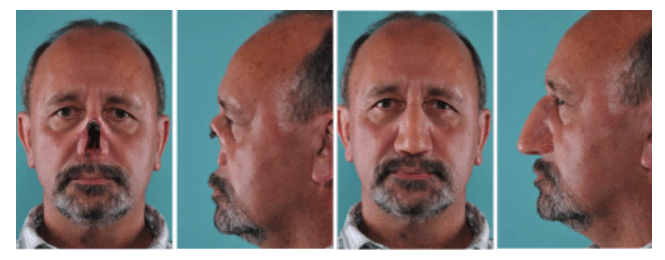

Figure 2: Post-nasectomy defect and prosthetic support. After radical resection of a massive squamous cell carcinoma (SCC) of the inferior nose, the patient was provided with an implant-supported silicone nasal prosthesis. 
Page 2 of 4

In August 2011, the patient presented to our department with a tumor recurrence at the base of the septum (Figure 3).

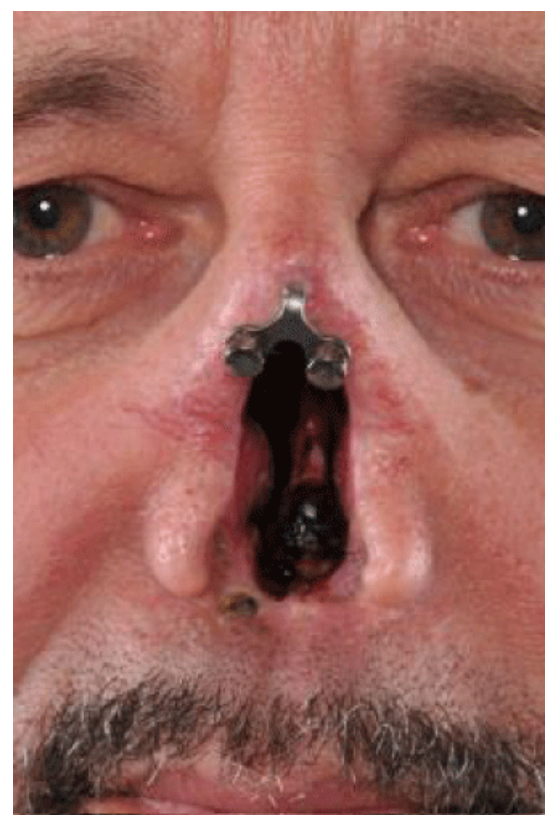

Figure 3: Recurrent SCC. Two years after the initial tumor resection, the patient presented with a tumor recurrence at the septal base.

The following mapping showed a moderately differentiated keratinizing SCC of the nasal floor.

In September 2011, the patient underwent partial nasectomy, resection of the septum, and partial maxillary resection performed by the maxillofacial surgery department of our hospital. After R0 resection and without evidence of lymph node or distant metastases, the same department carried out an augmentation of the previously resected maxilla using cortical bone from the iliac crest ten months later. Another five months later, elongation of the upper lip, removal of implants and nasal floor lining reconstruction with full-thickness skin grafting was performed (Figure 3).

In August 2013, four years after the initial presentation and two years after tumor recurrence, the patient presented again to our department for consultation with the defect seen below (Figure 4).
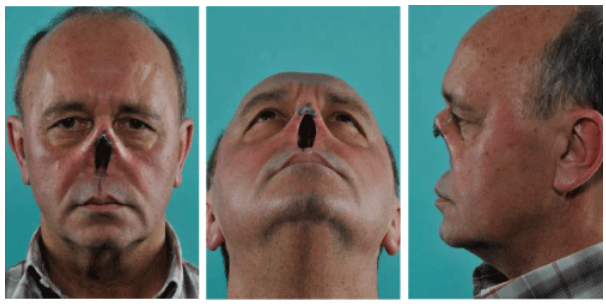

Figure 4: Extended post-nasectomy defect. After extended partial nasectomy, resection of the septum, partial maxillary resection and subsequent augmentation of the previously resected maxilla, the patient presented to our department with the defect seen on the left.
Thorough investigation of the patient's wishes yielded a strong tendency towards autologous reconstruction of the nose by the patient, currently provided with a silicone nasal prosthesis manufactured by the anaplastologist associated to our hospital (Figure 5).
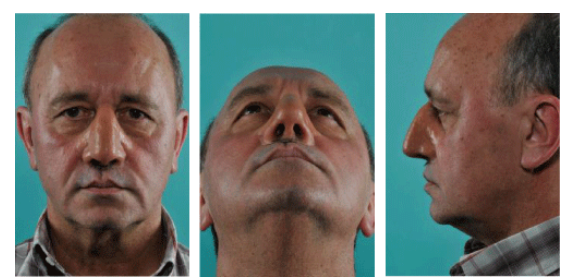

Figure 5: Prosthetic support. The patient wearing an implantsupported silicone nasal prosthesis manufactured by the anaplastologist associated to our hospital.

A reconstructive plan and timeline, spanning 6-12 months and including four to six operations were laid out to the patient, and informed consent was taken. In February 2014, after hair removal of the forearm donor site was completed, the first step of total nasal reconstruction was performed, using a left free radial forearm flap [3] for reconstruction of the inner lining of the nose. The flap was anastomosed micro surgically to the left superficial temporal artery and folded onto itself for intermediate outside coverage (Figure 6).
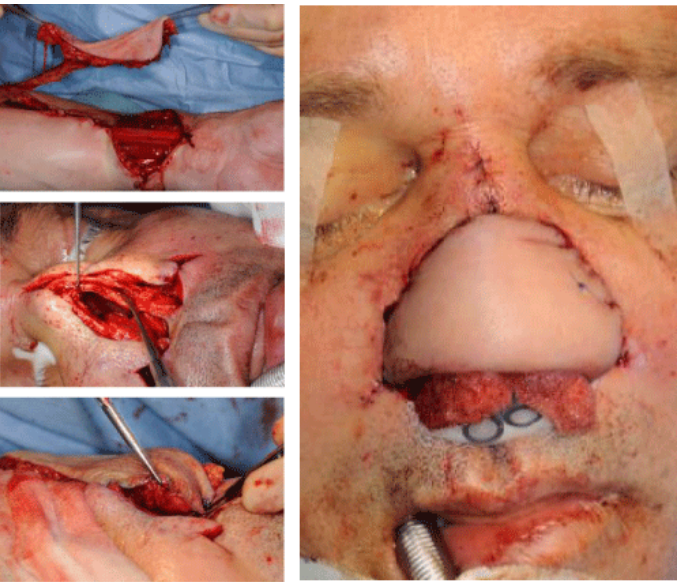

Figure 6: Inner lining reconstruction with a free flap. Harvesting and in setting of a free radial forearm flap for inner lining reconstruction. The flap is folded onto itself for intermediate outside coverage.

Additionally, rib cartilage from the right 9th rib was harvested, diced and filled into a bag of alloplastic fascia lata ("Diced Cartilage in Fascia") for further augmentation of the maxilla.

Two months later, reconstruction of the cartilaginous framework using rib cartilage [4] from the right 8 th rib and subsequent reconstruction of the nasal skin envelope using a right paramedian forehead flap [5] were carried out (Figure 7A and 7B). 


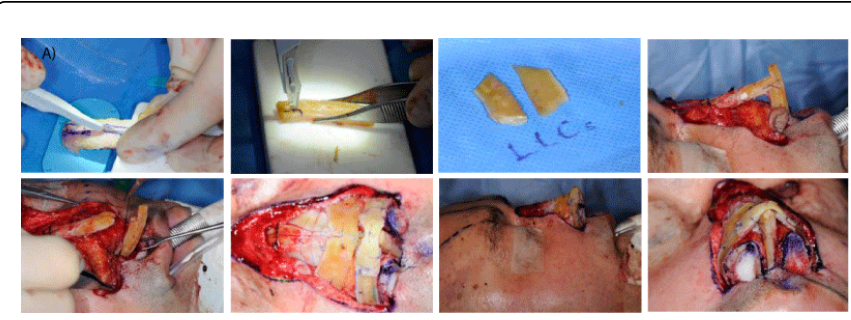

Figure 7A: Reconstruction of the cartilaginous framework. The nasal cartilaginous framework is reconstructed using rib cartilage from the right 8 th rib.
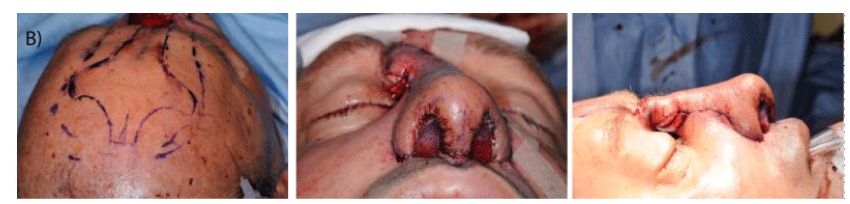

Figure 7B: Reconstruction of the nasal skin envelope. A right paramedian forehead flap is used for reconstruction of the nasal skin envelope.

In June 2014, the skin pedicle of the well perfused forehead flap was divided early, as skin from the pedicle was used for over-grafting a superficial necrosis on the left alar rim (Figure 8).

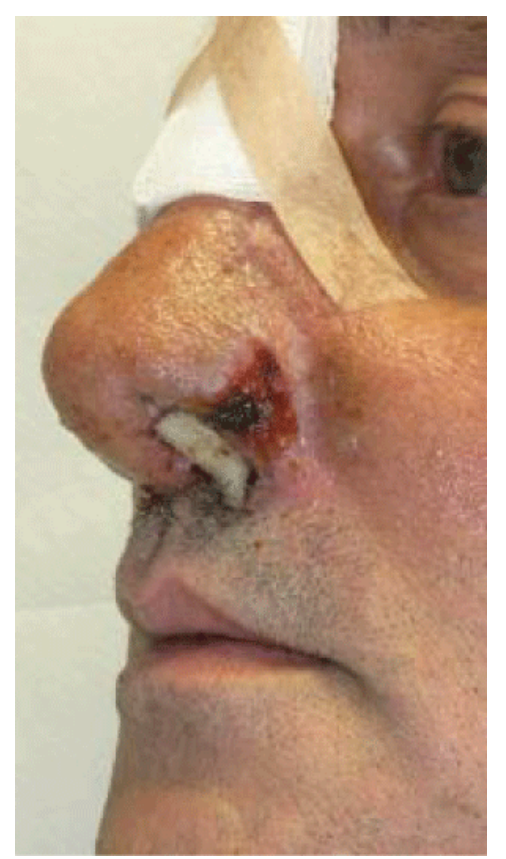

Figure 8: Superficial flap necrosis of the left ala. A superficial necrosis of the reconstructed skin of the left ala and alar rim, subsequently overgrafted with a full-thickness skin graft taken from the skin pedicle upon pedicle division.
Three and six months later, thinning of the left, respectively right alar was performed. The final result is seen in Figure 9.
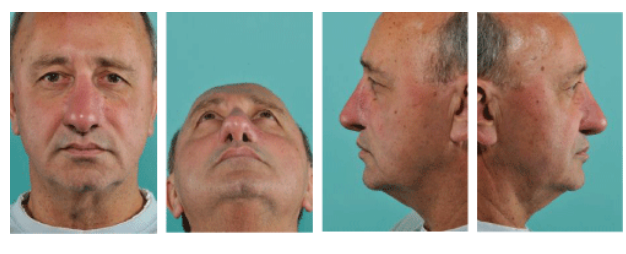

Figure 9: The final result. The autologous nose reconstruction is completed after 10 months. Photographs were taken 9 months after completion of the reconstruction.

\section{Discussion}

Central facial defects created by radical nasectomy are extremely mutilating, impairing not only the function of the nose, but also to a very significant degree patients' social lives and self-esteem. Therefore, adequate substitution of missing tissues, either by prosthesis, or by autologous reconstruction, must be the goal of any treatment plan. Form and function are the two main determinants of a successful nasal substitution or reconstruction. Anaplastology has made great leaps towards providing patients with a natural looking nose without impairing nasal function. Nasal prostheses can be supported by magnet-carrying implants, as well as by the defect itself, and are fitted to the patient and his/her defect individually. On the other hand, autologous reconstruction of the nose using free flaps for inner lining [3], rib cartilage for reconstruction of the framework [4], as well as a paramedian forehead flap for reconstruction of the skin envelope [5], has become the gold standard of nasal reconstruction [1,2], yielding very good and reliable results in terms of form and function. In our department, patients with post-nasectomy defects after excision of SCC are usually offered prosthetic support for two years to allow for early detection of any tumor recurrence. Once tumor-free, we offer autologous nasal reconstruction to most patients, excluding severely ill or elderly patients, keeping in mind that it is the patient's wish that determines any further action. If the patient wants to move away from prosthesis, an operative plan is worked out and laid out to the patient, usually consisting of four to six operative steps, covering 6-12 months. Informed consent is taken, and nasal reconstruction is carried out as in the case presented above.

Consisting of a very challenging series of operations, possibly leading to major complications such as flap necrosis or infection of the transplanted cartilaginous framework, autologous nasal reconstruction should only be considered if an outcome equivalent to prosthesis in terms of form and function can be expected. In our opinion, this major reconstructive surgery should only be performed by an experienced team in an adequate hospital setting. Our team conducts partial nasal reconstruction using the paramedian forehead flap about 65 times per year [6], and has, over the past years, performed 32 total nasal reconstructions using free flaps for inner lining. Complications such as cartilaginous infections have occurred, sometimes requiring revision surgery and even re-reconstruction of the framework after removal of the infected cartilage and recovery of the soft tissue, including salvage grafts and flaps. However, we feel that if the patient is willing and fit to undergo major reconstructive surgery of the nose, autologous reconstruction can be successfully carried out and a result superior to a prosthetic solution can be expected. 
Citation: Hoehne J and Fischer H (2016) Total Nasal Reconstruction after Nasectomy in a Case of Massive Squamous Cell Carcinoma of the Inferior Nose. Anaplastology 5: 158. doi:10.4172/2161-1173.1000158

Page 4 of 4

\section{Conclusion}

In the willing and fit patient with post-nasectomy defect, autologous reconstruction of the nose remains the gold standard therapy after a bridging period with a nasal prosthesis to allow for early detection of tumor recurrence. Consisting of a very challenging series of usually four to six operations, nasal reconstruction should only be performed by an experienced team in an adequate hospital setting.

\section{Conflict of Interest}

The authors declare that they have no conflict of interest as defined by the guidelines of the International Committee of Medical Journal Editors

\section{References}

1. Burget GC, Menick FJ (1994) Aesthetic reconstruction of the nose. St Louis.

2. Menick FJ (2008) Nasal Reconstruction: Art and Practice. SaundersElsevier.

3. Haack S, Fischer H, Gubisch W (2014) Lining in Nasal Reconstruction. Facial Plast Surg 30: 287-299.

4. von Gregory H, Fischer H, Eppstein RJ, Gubisch W (2014) Framework Fabrication with Rib Cartilage in Partial and Total Nasal Reconstruction. Facial Plast Surg 30: 306-317.

5. Fischer H (2014) Nasal Reconstruction with the Paramedian Forehead Flap - Details for Success, Facial Plast Surg 30: 318-331.

6. Fischer H, Gubisch W (2008) Nasal reconstruction: a challenge for plastic surgery. Dtsch Arztebl Int 105: 741-746. 